\title{
More than Meets the Eye: An Interesting Case of Cardiac
}

\section{Tamponade}

\section{Clarke CA*}

Internal Medicine physician, Loma Linda University Health, USA

*Corresponding author: Camille A Clarke, MD, FACP, Division of Internal and Preventive Medicine, Loma Linda University Health, 24785 Stewart St., Evans Hall Suite 204, Loma Linda, CA 92350, USA; Email: cclarke@llu.edu

\section{Case Report}

Volume 2 Issue 2

Received Date: June 20, 2019

Published Date: August 09, 2019

DOI: $10.23880 /$ aabsc- 16000137

\section{Abstract}

This interesting case highlights the presentation of a 68-year-old Latino male with coronary artery disease and coronary artery bypass graft surgery eight years who presented with non-specific respiratory symptoms. At the time of his initial evaluation he was noted to have persistent bigeminy and a 5 beat run of ventricular tachycardia. During his hospital stay he was again noted to have marked arrhythmias, with a 40 beat run of ventricular tachycardia. Urgent echocardiogram was obtained and revealed significant pericardial effusion compressing his right ventricle. On cardiac catheterization patient was found to have a patent graft but trasudative pericardial fluid causing tamponade and arrhythmia both which completely resolved after drainage.

Learning Objective 1: Recognize ventricular arrhythmias as an atypical presentation of pericardial effusion and cardiac tamponade.

Learning Objective 2: Appreciate the utility of echocardiogram in elucidating etiologies of non-sustained ventricular tachycardia.

Keywords: Pericardial effusion; Bigeminy; NSVT; Echocardiogram; Coronary artery disease

\section{Case Report}

A 68 year old Latino male with coronary artery disease and coronary artery bypass graft surgery eight years prior presented with symptoms of progressive exertional shortness of breath for the past three months with acute worsening in the preceding three days. He endorsed mild palpitations during these episodes but denied any accompanying chest pain, orthopnea, dizziness, syncope or abnormal constitutional symptoms. On arrival, he was noted to have persistent bigeminy with a 5 beat run of ventricular tachycardia. Vitals signs were within normal and electrocardiogram revealed sinus rhythm with persistent bigeminy but no ST-T segment changes. His basic metabolic panel was unremarkable, troponin negative and hematocrit 38. Chest x-ray showed minimal enlarged heart with no lung pathology. Physical exam revealed jugular venous distention to the angle of his mandible, lungs were clear and cardiac exam normal. On the night of admission patient had persistent bigeminy with significant couplets and a 40 beat run of ventricular tachycardia. He remained hemodynamically stable throughout. Echocardiogram was performed and showed inferoapical wall akinecity and two pockets of pericardial effusion behind the left atrium as well as a compressed right ventricle. He was transferred for cardiac 
catheterization, which revealed a patent cardiac graft but transudative pericardial fluid causing tamponade, which resolved after drainage.

\section{Discussion}

Although ventricular arrhythmias have been reported as a presenting manifestation of metastatic or infectious cardiac arrhythmias it is an uncommon presenting symptom of transudative pericardial effusion [1]. Cardiac tamponade, a life-threatening condition, is caused by a slow or rapid compression of the heart due to pericardial accumulation and intrapericardial pressure [2,3]. Classic findings such as distant heart sounds, pulsus paradoxus, elevated jugular venous pressure are helpful in diagnosis and chest imaging revealing cardiomegaly or electrocardiographic changes can help direct clinicians towards this diagnosis [4-6]. These signs and symptoms are not always present, however, and their absence could be misleading thus a high index of suspicion should be maintained in certain clinical contexts $[7,8]$. Our patient had a history of pericarditis and a pocket of pericardial fluid that accumulated and compressed his right ventricle, leading to cardiac tamponade with resultant ventricular arrhythmias [9]. It is very likely that, in addition to the effusion, the stiffness of his pericardium from prior disease contributed to this large relative compression. Though ischemia was an appropriate initial suspicion given his history of coronary artery bypass graft surgery and cardiac risk factors, timely echocardiogram was imperative. Subcostal four-chamber view revealed both the size and location of the effusion, which was causing significant chamber compression that resolved once the effusion was drained [10] (Figures 1 \& 2).

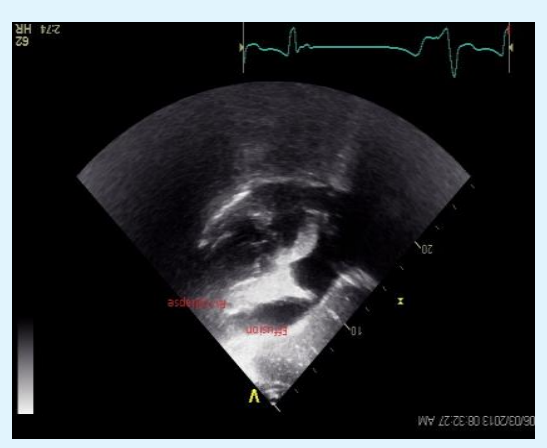

Figure 1: Subcostal view showing RV collapse and pericardial effusion causing ventricular arrythmia.

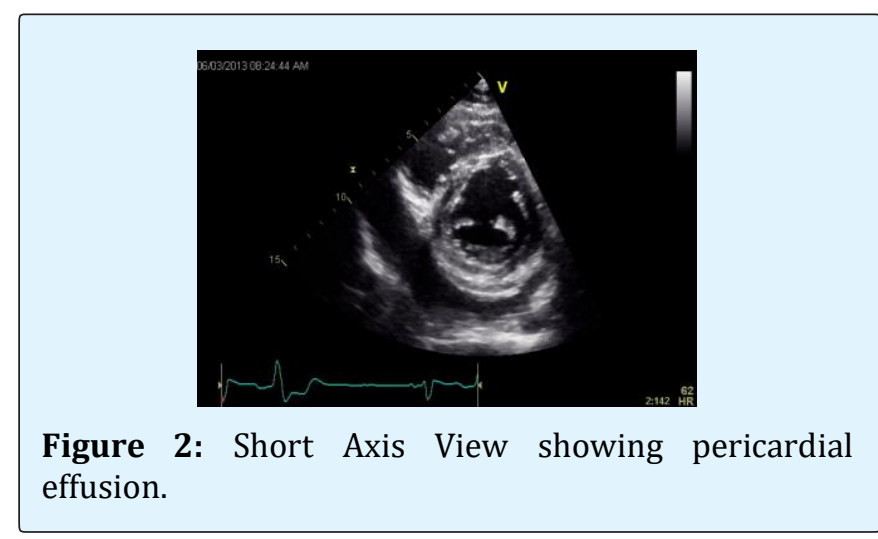

\section{Conclusion}

For patients with history of pericarditis, the appearance of ventricular arrhythmias should raise the suspicion for cardiac tamponade. Cases may go undiagnosed because typical associated clinical findings are absent or unapparent thus timely identification through emergent echocardiography plays a key role in confirming the diagnosis.

\section{Conflicts and Competing Interests: None.}

Acknowledgement: Dr. Deepak Thatai, board certified cardiologist and chief of Cardiology at Cambridge Health Alliance.

\section{References}

1. Spodick DH (2001) Pericardial diseases. In: Braunwald E, Zipes DP, Libby P, (Eds.), Heart disease: a textbook of cardiovascular medicine, $6^{\text {th }}$ (Edn.), WB. Saunders, Philadelphia, pp: 1823-1876.

2. Reddy PS, Curtiss EI, Uretsky BF (1990) Spectrum of hemodynamic changes in cardiac tamponade. Am J Cardiol 66(20): 1487-1491.

3. Beloucif S, Takata M, Shimada M, Robotham JL (1992) Influence of pericardial constraint on atrioventricular interactions. Am J Physiol 263: H125-H134

4. Spodick DH (2003) Acute cardiac tamponade. N Engl J Med 349: 684-690.

5. Bilchick KC, Wise RA (2002) Paradoxical physical findings described by Kussmaul: pulsus paradoxus and Kussmaul's sign. Lancet 359(9321): 1940-1942. 
6. Swami A, Spodick DH (2003) Pulsus paradoxus in cardiac tamponade: a pathophysiologic continuum. Clin Cardiol 26(5): 215-217.

7. Sharma N, Panchal V, Kalaria VG (2004) A typical hemodynamic manifestations of cardiac tamponade. Catheter Cardiovasc Interv 63(3): 339-345

8. Ariyarajah V, Spodick DH (2007) Cardiac tamponade revisited: a postmortem look at a cautionary case. Tex Heart Inst J 34(3): 347-351
9. Santamore WP, Li KS, Nakamoto T, Johnston WE (1990) Effects of increased pericardial pressure on the coupling between the ventricles. Cardiovasc Res 24(9): 768-776.

10. D’Cruz I, Rehman AU, Hancock HI (1997) Quantitative echocardiographic assessment in pericardial disease. Echocardiography 14(2): 207-214. 